\title{
Feasibility, Safety, and Long-Term Outcomes of Zero-Contrast Percutaneous Coronary Intervention in Patients With Chronic Kidney Disease
}

\author{
Keita Shibata, MD; Kohei Wakabayashi, MD, PhD; Tomoyuki Ishinaga, MD; \\ Mitsuyuki Morimura, MD; Naoki Aizawa, MD; Toshiaki Suzuki, MD; \\ Takahiro Furuya, MD, PhD; Chisato Sato, MD, PhD; Tenjin Nishikura, MD, PhD; \\ Naoko Ikeda, MD, PhD; Miwa Kikuchi, MD, PhD; Kaoru Tanno, MD, PhD; \\ Toshiro Shinke, MD, PhD; Masahiko Izumizaki, MD, PhD
}

\begin{abstract}
Background: The long-term safety and utility of intravascular ultrasound (IVUS)-guided zero-contrast percutaneous coronary intervention $(\mathrm{PCl})$ in patients with chronic kidney disease (CKD) are unknown.

Methods and Results: A total of 698 consecutive patients treated with $\mathrm{PCl}(1,061$ procedures) in our center were studied. Patients with acute coronary syndrome, who are on maintenance hemodialysis, and who had a planned rotational atherectomy were excluded. Finally, they were divided into 2 groups: zero-contrast $\mathrm{PCI}(\mathrm{n}=55,78$ procedures) and conventional $\mathrm{PCl}(\mathrm{n}=462,670$ procedures). After propensity score matching, 50 patients were matched for each group to evaluate long-term outcomes. Primary endpoints were major adverse cardiovascular events (MACE), including all-cause death, non-fatal myocardial infarction (MI), and clinically driven target lesion revascularization. All patients in the zero-contrast $\mathrm{PCl}$ group had stage 3-5 CKD with an estimated glomerular filtration rate of $38.3 \pm 14.8 \mathrm{~mL} / \mathrm{min} / 1.73 \mathrm{~m}^{2}$. Zero-contrast $\mathrm{PCl}$ was successful in all 78 procedures without renal events such as acute kidney injury or emergent hemodialysis and procedural complications such as coronary perforation or periprocedural MI. During a follow-up period of 32 months, 7 patients died ( 1 cardiac, 6 non-cardiovascular), and 4 patients were introduced to renal replacement therapy. The incidence of MACE was similar between the zero-contrast and conventional PCl groups (log-rank, P=0.95).
\end{abstract}

Conclusions: IVUS-guided zero-contrast PCI might be safe and feasible in patients with CKD with satisfactory acute and long-term renal and cardiovascular outcomes.

Key Words: Chronic kidney disease; Contrast media; Intravascular ultrasound; Percutaneous coronary intervention

I $t$ is well known that patients with chronic kidney disease (CKD) have a high cardiovascular event rate. ${ }^{1-4}$ The recent ISCHEMIA-CKD trial demonstrated that an initial invasive strategy did not improve the clinical outcomes in patients with moderate or severe ischemic heart disease and advanced CKD compared with an initial conservative strategy. ${ }^{5}$ One explanation may be that most acute coronary syndrome cases occur as a consequence of plaque rupture in arteries without high-grade stenosis. Thus, prevention of acute coronary syndrome by prophylactic revascularization with percutaneous coronary intervention (PCI) is often difficult. ${ }^{6}$ The more important cause may be contrast use during the PCI procedure. CKD is the strongest predictor of contrast-associated acute kidney

\section{Editorial p????}

injury (AKI) development. Many studies have demonstrated that worsening renal function due to contrast use is strongly associated with cardiovascular events. ${ }^{7-9}$ It is also known that an under-expanding stent is associated with target vessel failure after PCI. ${ }^{10,11}$ Thus, a high-quality imaging-guided PCI procedure with less contrast volume should improve the clinical outcomes of patients with $\mathrm{CKD}$ and ischemic heart disease. There is some evidence that mini-contrast PCI prevents renal events; ${ }^{\mathbf{1 2}-16}$ however, little data are available on the feasibility and safety of zerocontrast PCI for patients with CKD, ${ }^{17,18}$ and the long-term

Received October 27, 2021; revised manuscript received December 30, 2021; accepted January 16, 2022; J-STAGE Advance Publication released online February 10, 2022 Time for primary review: 14 days

Division of Cardiology, Cardiovascular Center, Showa University Koto-Toyosu Hospital, Tokyo (K.S., K.W., T.I., M.M., N.A., T. Suzuki, T.F., C.S., T.N., N.I., M.K., K.T.); Department of Physiology, Showa University School of Medicine, Tokyo (K.S., M.I.); and Division of Cardiology, Showa University Hospital, Tokyo (T. Shinke), Japan

Mailing address: Kohei Wakabayashi, MD, PhD, FJCS, FACC, Division of Cardiology, Cardiovascular Center, Showa University Koto-Toyosu Hospital, 5-1-38 Toyosu, Koto-ku, Tokyo 135-8577, Japan. E-mail: koheiw@med.showa-u.ac.jp

All rights are reserved to the Japanese Circulation Society. For permissions, please e-mail: cj@j-circ.or.jp

ISSN-1346-9843 
outcomes are unknown. ${ }^{19}$ We hypothesized that optimal revascularization by intravascular ultrasound (IVUS)guided zero-contrast PCI is safe and provides satisfactory long-term outcomes. The present study was conducted to determine the acute and long-term renal and cardiovascular outcomes in patients with CKD who underwent IVUSguided zero-contrast PCI.

\section{Methods}

\section{Study Population and Design}

The study cohort was from a single-center PCI registry designed to collect data on patient characteristics, procedures, and acute and long-term clinical outcomes. We performed a retrospective analysis to assess the feasibility and safety of zero-contrast PCI with the data from the allcomer registry. The indication for elective PCI was decided for all patients by the heart team conference consisting of interventional cardiologists, surgeons, general cardiologists, and general physicians at Showa University KotoToyosu Hospital. Patients with chronic coronary syndrome (CCS) and stage 3-5 CKD (estimated glomerular filtration rate $[\mathrm{eGFR}]<60 \mathrm{~mL} / \mathrm{min} / 1.73 \mathrm{~m}^{2}$ by the Modification of Diet in Renal Disease equation and abnormal serum creatinine) have undergone zero-contrast PCI at our institute since October 2015. Other patients were treated with conventional PCI using contrast agents. IVUS-guided PCI was performed for not only zero-contrast PCI but also for conventional PCI. Patients who underwent emergent PCI for acute coronary syndrome were excluded from the study. Other exclusion criteria were a history of maintenance hemodialysis and planned rotational atherectomy. Patients were enrolled between October 2015 and December 2018. Clinical outcomes were assessed until December 2020. Informed consent for the PCI procedure included the benefits, risks of revascularization, and details of the zero-contrast PCI strategy. All enrolled patients provided written informed consent for their PCI strategy before the procedure. If the patient did not consent to zero-contrast PCI, the patient was treated with mini-contrast PCI. Informed consent for data use in the present study was obtained in the form of an opt-out. The study was conducted in accordance with the Declaration of Helsinki and approved by the ethics committee of Showa University Koto-Toyosu Hospital.

The efficacy and safety of zero-contrast PCI were evaluated in the short and long term, respectively. Short-term endpoints included success of zero-contrast PCI and complications such as coronary perforation, stent thrombosis, and periprocedural myocardial infarction (MI), which was defined as type 4a MI according to the American College of Cardiology/American Heart Association/European Society of Cardiology universal definition of MI. ${ }^{20}$ Successful zero-contrast PCI was defined as optimal revascularization with a minimum stent area $>90 \%$ of the mean of the proximal and distal stent reference, and no contrast use until final assessment. Successful conventional PCI was defined as optimal revascularization with a minimum stent area $>90 \%$ of the mean of the proximal and distal stent reference, regardless of the volume of contrast use. Longterm endpoints were major cardiovascular events (MACEs), including all-cause death, non-fatal MI, and clinically driven target lesion revascularization. To determine the long-term safety and efficacy of zero-contrast PCI, the incidence of MACE was compared with the control group, which was propensity score matched, as explained below.
The other endpoints were renal function and events. Variation in renal function was assessed using the serum creatinine level 1 day before and 1 day, 1 month, and 1 year after PCI. The incidence of renal replacement therapy was also reviewed over the short-and long-term clinical course.

\section{Procedural Protocol}

Intravenous hydration saline $(1 \mathrm{~mL} / \mathrm{kg} / \mathrm{h})$ was administered for $12 \mathrm{~h}$ before and after the procedure for both coronary angiography and PCI. A biplane cine system was used to reduce the contrast volume in patients with $\mathrm{CKD}$ who underwent coronary angiography. Angiography images were taken with iso-osmolar contrast medium using a 4Fr diagnostic catheter, 1 injection of contrast $(4 \mathrm{~mL})$ with 2 directions for the right coronary artery, and 2 injections of contrast $(5 \mathrm{~mL})$ with 4 directions for the left coronary artery. Thus, a total of $14 \mathrm{~mL}$ of contrast was used for coronary angiography. Further injection was added if necessary for coronary tree assessment; however, the contrast was not diluted. The patients underwent elective PCI for at least 10 days after coronary angiography. Patients receiving unfractionated heparin were given a bolus of $100 \mathrm{U} / \mathrm{kg}$, and additional unfractionated heparin was administered to achieve an activated clotting time $>250 \mathrm{~s}$ if necessary. All patients received dual antiplatelet agents, as appropriate. Access, such as the radial or femoral approach, was left to the discretion of the operator. The previous coronary angiography image was referred to for PCI guidance. The guiding catheter was engaged without testing by contrast injection. The wire was first crossed with the target lesion. If the patient had a bifurcation lesion requiring protection of the side branch or the branches were used as landmarks, additional guide wires were placed. PCI was performed under IVUS guidance. The procedure strategy was decided upon based on the IVUS findings, such as plaque morphology, vessel size, lesion length, and relationship to the side branch. The proximal and distal points of the target lesion were marked by an IVUS transducer in dry cine angiograms in 2 directions using a biplane system. Previously deployed stents or coronary calcifications were also referred to as landmarks. These markers were referred to for the location of balloon dilatation and stent deployment. IVUS was repeated as required during the procedure. Angiography using contrast was not permitted even if the patient had chest pain or ST changes on an electrocardiogram. The optimal procedure or drug administration was performed after IVUS evaluation of blood flow, dissection, hematoma, and main or branch occlusion. The procedure used for further serious complications depended on the operator's discretion. All patients received drug-eluting stents during the study period. A final IVUS was performed to assess coronary flow, branch occlusion, stent expansion and apposition, dissection, hematoma, and other complications. ${ }^{21,22}$ Zero-contrast PCI was defined as PCI without contrast until the final IVUS assessment of the procedure. Final angiography was performed with $2-6 \mathrm{~mL}$ of contrast to assess the safety of the procedure, such as wire perforation, distal embolization, or dissection, which cannot be detected on IVUS and transthoracic echocardiography. We have previously published the details of the PCI strategy without contrast. $^{23}$

\section{Statistical Analysis}

Categorical variables are presented as frequencies and percentages and compared between groups using a chi-squared 


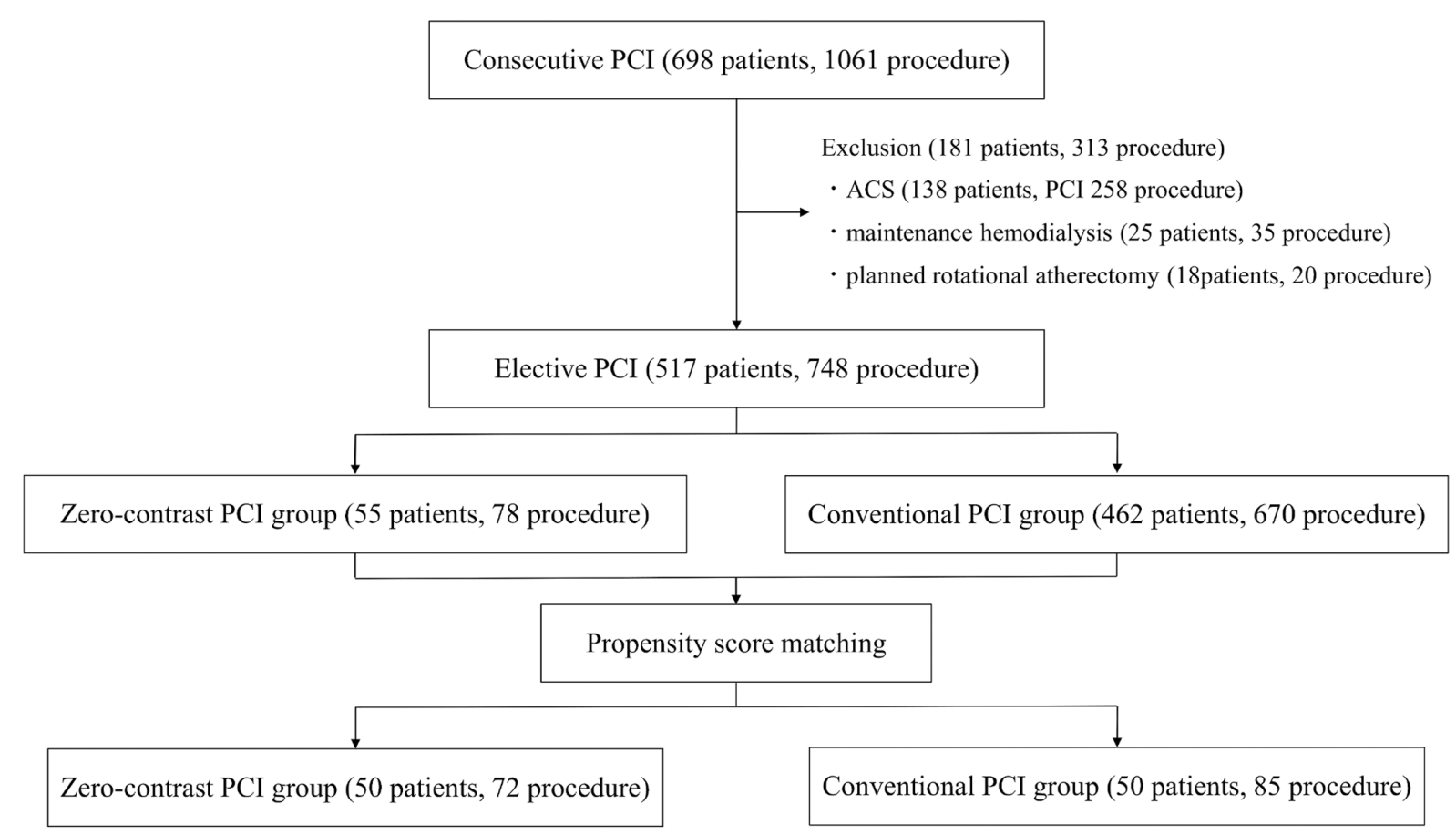

Figure 1. A total of 698 patients underwent percutaneous coronary intervention (PCl) at our center between October 2015 and December 2018. After excluding patients with acute coronary syndrome (ACS), those requiring maintenance hemodialysis, and those who had a planned rotational atherectomy, there were 517 patients and 748 elective PCls. A total of 55 patients consented to the zero-contrast $\mathrm{PCl}$ strategy, and 78 elective $\mathrm{PCl}$ procedures were performed. The remaining 462 patients underwent conventional $\mathrm{PCl}$. Based on propensity scores, 50 patients were matched for each group.

or Fisher's exact test, as appropriate. Continuous variables are presented as the mean \pm standard deviation (SD) or median and interquartile range (IQR) for skewed data, and compared between groups using 2-tailed, unpaired t-tests or, if parameters were not normally distributed, using a Mann-Whitney U-test. Serum creatinine levels were compared between pre-procedure and the next day, 1 month, or 1 year after the procedure using a Wilcoxon test. Oneto-one propensity score matching was used to reduce the effect of clinical background between the zero-contrast and conventional PCI groups. Propensity score matching was estimated using a non-parsimonious logistic regression model for conventional vs. zero-contrast PCI. To calculate the propensity score, the following variables were included in the analysis: sex, age, smoking, diabetes, hypertension, dyslipidemia, history of cerebrovascular disease, history of heart failure, prior PCI, prior coronary artery bypass graft, and left ventricular ejection fraction. The Hosmer and Lemeshow goodness-of-fit test was used to assess how the model fitted to the data. The caliper width was set as 0.05 $\mathrm{SD}$ of the PS logit. Incidences of long-term outcomes after the index PCI were estimated using the Kaplan-Meier method, and the difference between the 2 groups was assessed using a log-rank test. Statistical significance was set at $\mathbf{P}<0.05$. Statistical analyses were performed using JMP Pro version 15.0 software (SAS Institute Inc., Cary, NC, USA).

\section{Results}

A total of 698 patients underwent PCI at our center between
October 2015 and December 2018 (Figure 1). From these consecutive patients, emergent PCI, history of maintenance hemodialysis, or planned rotational atherectomy cases were excluded. A total of 517 patients underwent elective PCI (748 procedures). There were 5 patients with stage 5 CKD and 8 patients with stage $4 \mathrm{CKD}$, all of whom underwent zero-contrast PCI. Among the 66 patients with stage 3 CKD, 42 underwent zero-contrast PCI. Thus, a total of 55 patients consented to the zero-contrast PCI strategy, and 78 elective PCI procedures were performed without contrast for these patients. Twenty-four patients with stage 3 CKD chose mini-contrast PCI and those patients were included to conventional PCI group. The remaining 462 patients underwent conventional PCI.

The clinical characteristics of 55 patients who underwent zero-contrast PCI and 462 patients who underwent conventional PCI are shown in Table 1. The age was similar between the 2 groups. The patients from the zero-contrast PCI group were more frequently male and had diabetes mellitus, and a history of cardiovascular disease such as heart failure and stroke. Lower hemoglobin and higher B-type natriuretic peptide were observed in the zero-contrast PCI group. All patients in the zero-contrast PCI group had stage 3-5 CKD with a median creatinine level of $1.38 \mathrm{mg} / \mathrm{dL}$ (1.15-1.76), and an eGFR of $38.3 \pm 14.8 \mathrm{~mL} / \mathrm{min} / 1.73 \mathrm{~m}^{2}$. There were some differences in medication at discharge.

After propensity score matching between the 55 patients treated with zero-contrast PCI and 462 patients treated with conventional PCI, 50 patients were matched for each group. The chi-squared test statistic was $8.13(\mathrm{P}=0.77)$, 


\begin{tabular}{|c|c|c|c|}
\hline Characteristics & $\begin{array}{c}\text { Conventional } \mathrm{PCl} \\
(\mathrm{n}=462)\end{array}$ & $\begin{array}{c}\text { Zero-contrast PCl } \\
(n=55)\end{array}$ & $P$ value \\
\hline Age, years & $70.9 \pm 11.5$ & $72.5 \pm 12.0$ & 0.32 \\
\hline Female & $117(25.3)$ & $7(12.7)$ & 0.039 \\
\hline History of smoking & $298(64.5)$ & $39(70.9)$ & 0.35 \\
\hline Diabetes mellitus & $176(38.1)$ & $32(58.2)$ & 0.0041 \\
\hline Hypertension & $310(67.1)$ & $41(74.5)$ & 0.26 \\
\hline Hyperlipidemia & $282(61.0)$ & $33(60.0)$ & 0.88 \\
\hline History of heart failure & $42(9.1)$ & $17(30.9)$ & $<0.0001$ \\
\hline Previous $\mathrm{PCl}$ & $134(29.0)$ & $15(27.2)$ & 0.79 \\
\hline Previous CABG & $2(0.43)$ & $3(5.5)$ & 0.0098 \\
\hline Prior Ml & $80(17.3)$ & $13(23.6)$ & 0.25 \\
\hline History of CVD & $48(10.4)$ & $14(25.5)$ & 0.0012 \\
\hline LVEF, \% & $59.1 \pm 11.6$ & $56.1 \pm 13.8$ & 0.074 \\
\hline \multicolumn{4}{|l|}{ Laboratory data } \\
\hline Hemoglobin, g/L & $13.0 \pm 1.79$ & $12.0 \pm 2.09$ & $<0.0001$ \\
\hline Creatinine, $\mathrm{mg} / \mathrm{dL}$ & $0.80(0.69-0.92)$ & $1.38(1.15-1.76)$ & $<0.0001$ \\
\hline eGFR, $\mathrm{mL} / \mathrm{min} / 1.73 \mathrm{~m}^{2}$ & $70.6 \pm 17.6$ & $38.3 \pm 14.8$ & $<0.0001$ \\
\hline $\mathrm{BNP}, \mathrm{pg} / \mathrm{mL}$ & $46.3(18.9-98.4)$ & $120.9(27.5-249.7)$ & 0.0002 \\
\hline LDL-cholesterol, mg/dL & $101 \pm 31.6$ & $95.8 \pm 32.1$ & 0.31 \\
\hline HDL-cholesterol, mg/dL & $47.9 \pm 14.1$ & $46.9 \pm 17.3$ & 0.69 \\
\hline Triglyceride, mg/dL & $171.9 \pm 139.1$ & $141.7 \pm 72.9$ & 0.11 \\
\hline Hemoglobin A1c, \% & $6.27 \pm 1.05$ & $6.49 \pm 1.27$ & 0.24 \\
\hline \multicolumn{4}{|l|}{ Lesion severity } \\
\hline 3-vessel disease & $16(3.5)$ & $5(9.1)$ & 0.061 \\
\hline 2-vessel disease & $138(29.9)$ & $19(34.6)$ & 0.48 \\
\hline 1-vessel disease & $308(66.7)$ & $31(56.4)$ & 0.13 \\
\hline Left main trunk disease & $22(8.3)$ & $6(11.0)$ & 0.10 \\
\hline \multicolumn{4}{|l|}{ Medication at discharge } \\
\hline Aspirin & 406 (87.9) & $46(81.8)$ & 0.20 \\
\hline P2Y12 inhibitors & 457 (98.9) & $54(98.2)$ & 0.49 \\
\hline Anticoagulant & $65(14.1)$ & $11(20.0)$ & 0.24 \\
\hline Statin & $336(72.7)$ & $38(69.1)$ & 0.57 \\
\hline ACE-i or ARB & 226 (48.9) & $30(54.6)$ & 0.43 \\
\hline$\beta$-blocker & $157(34.0)$ & $27(49.1)$ & 0.027 \\
\hline Calcium blocker & $206(44.6)$ & $32(58.2)$ & 0.056 \\
\hline Diuretics & 89 (19.3) & $25(45.5)$ & $<0.0001$ \\
\hline
\end{tabular}

Data are presented as $n(\%)$, mean \pm SD, and/or median (Q1-Q3). ACE-i, angiotensin-converting-enzyme inhibitor; ARB, angiotensin II receptor blocker; BNP, B-type natriuretic peptide; CABG, coronary artery bypass grafting; CVD, cerebrovascular disease; eGFR, estimated glomerular filtration rate; HDL, high-density lipoprotein; LDL, low-density lipoprotein; LVEF, left ventricular ejection fraction; MI, myocardial infarction; PCl, percutaneous coronary intervention.

which indicates a good fit. The c-statistic for the model was 0.763 , indicating good discrimination. A comparison of clinical and angiographical characteristics between the 2 groups is shown in Tables 2 and 3. The clinical characteristics were well balanced, except the parameters regarding CKD. The patients from the zero-contrast PCI group more frequently had calcified lesions and tended to have more complexed lesions. The radiation duration was longer in the zero-contrast PCI group than in the conventional PCI group $(\mathrm{P}=0.046)$; however, the radiation dose was lower in the zero-contrast PCI group $(\mathrm{P}<0.0001)$. Minimum lumen area before PCI and minimum stent area after PCI were comparable between the 2 groups.

There were 15 patients in the conventional PCI group and 13 patients in the zero-contrast PCI group who underwent PCI with a trans-femoral approach. The main reason of the trans-femoral approach was large guiding catheter use more than $7 \mathrm{Fr}$ for bifurcation lesions, especially in the left main trunk, or a chronic total occlusion (CTO) lesion (13 patients in the conventional PCI group and 11 patients in the zero-contrast PCI group). Another reason was difficulty of radial artery access because of a curved artery or occlusion. Access was impossible from the radial artery in 2 patients in each group.

The in-hospital and long-term outcomes are summarized in Table 4. The median follow-up period was 1,083 (437$1,460)$ days in the zero-contrast PCI and $909(390-1,340)$ days in the conventional PCI groups. There was no failurePCI and no procedural complications such as persistent coronary dissection or hematoma, peri-procedural MI, or wire perforation in the zero-contrast PCI group. In the follow-up period, 8 and 7 patients experienced MACE in 


\begin{tabular}{|c|c|c|c|}
\hline Characteristics & $\begin{array}{c}\text { Conventional PCl } \\
(n=50)\end{array}$ & $\begin{array}{l}\text { Zero-contrast PCl } \\
\quad(n=50)\end{array}$ & $P$ value \\
\hline Age, years & $70.7 \pm 12.0$ & $72.3 \pm 12.4$ & 0.52 \\
\hline Female & $6(12.0)$ & $7(14.0)$ & 0.76 \\
\hline History of smoking & $36(72.0)$ & $34(68.0)$ & 0.66 \\
\hline Diabetes mellitus & $29(58.0)$ & $27(54.0)$ & 0.69 \\
\hline Hypertension & $34(68.0)$ & $36(72.0)$ & 0.66 \\
\hline Hyperlipidemia & $34(68.0)$ & $29(58.0)$ & 0.30 \\
\hline History of heart failure & $14(28.0)$ & $17(34.0)$ & 0.52 \\
\hline Previous $\mathrm{PCl}$ & $13(26.0)$ & $12(24.0)$ & 0.82 \\
\hline Previous CABG & $1(2.0)$ & $0(0)$ & 1.00 \\
\hline Prior MI & $12(24.0)$ & $11(22.0)$ & 1.00 \\
\hline History of CVD & $15.0(30.0)$ & $12(24.0)$ & 0.50 \\
\hline LVEF, \% & $57.2 \pm 12.8$ & $57.0 \pm 13.1$ & 0.93 \\
\hline \multicolumn{4}{|l|}{ Laboratory data } \\
\hline Hemoglobin, g/L & $13.1 \pm 1.61$ & $12.1 \pm 2.14$ & 0.0067 \\
\hline Creatinine, $\mathrm{mg} / \mathrm{dL}$ & $0.83(0.72-0.94)$ & $1.38(1.12-1.73)$ & $<0.0001$ \\
\hline eGFR, $\mathrm{mL} / \mathrm{min} / 1.73 \mathrm{~m}^{2}$ & $72.6 \pm 13.6$ & $38.9 \pm 15.2$ & $<0.0001$ \\
\hline BNP, pg/mL & $51.0(20.2-149.6)$ & $100.2(26.4-232.0)$ & 0.16 \\
\hline LDL-cholesterol, mg/dL & $101.8 \pm 36.5$ & $98.0 \pm 32.5$ & 0.73 \\
\hline HDL-cholesterol, mg/dL & $44.6 \pm 14.3$ & $46.3 \pm 17.5$ & 0.76 \\
\hline Triglyceride, mg/dL & $138.4 \pm 83.3$ & $147.8 \pm 73.5$ & 0.71 \\
\hline Hemoglobin A1c, \% & $6.85 \pm 1.53$ & $6.47 \pm 1.30$ & 0.40 \\
\hline \multicolumn{4}{|l|}{ Lesion severity } \\
\hline 3-vessel disease & $6(12.0)$ & $5(10.0)$ & 1.0 \\
\hline 2-vessel disease & $17(34.0)$ & $18(36.0)$ & 0.83 \\
\hline 1-vessel disease & $27(54.0)$ & $27(54.0)$ & 1.0 \\
\hline Left main trunk disease & $1(2.0)$ & $6(12.0)$ & 0.11 \\
\hline \multicolumn{4}{|l|}{ Medication at discharge } \\
\hline Aspirin & $44(88.0)$ & $41(82.0)$ & 0.40 \\
\hline P2Y12 inhibitors & $49(98.0)$ & $50(100.0)$ & 1.00 \\
\hline Anticoagulant & $6(2.0)$ & $9(4.0)$ & 0.58 \\
\hline Statin & $41(82.0)$ & $34(68.0)$ & 0.11 \\
\hline ACE-i or ARB & $24(48.0)$ & $28(56.0)$ & 0.42 \\
\hline$\beta$-blocker & $18(36.0)$ & $24(48.0)$ & 0.22 \\
\hline Calcium blocker & $21(42.0)$ & $30(60.0)$ & 0.072 \\
\hline Diuretics & $19(38.0)$ & $21(42.0)$ & 0.68 \\
\hline
\end{tabular}

Data are presented as $\mathrm{n}(\%)$, mean $\pm \mathrm{SD}$, and/or median (Q1-Q3). Abbrevitions as in Table 1.

the zero-contrast and conventional PCI groups, respectively. There was no significant difference in the KaplanMeier curves for MACE-free survival between the 2 groups (log-rank, $\mathrm{P}=0.95$; Figure 2).

In 55 patients who underwent zero-contrast PCI, renal events such as AKI and the requirement for emergent hemodialysis did not occur during hospitalization. In terms of long-term outcomes, 7 patients died (1 cardiac, 6 non-cardiovascular) and 4 patients were introduced to renal replacement therapy. Dialysis was performed in 3 patients with stage 5 CKD $(60.0 \%)$ and in 1 patient with stage 4 CKD $(12.5 \%)$ during the long-term follow-up period.

Variations in renal function assessed by serum creatine level are shown in Figure 3. In the conventional PCI group, serum creatinine levels tended to increase at 1 month and 1 year compared with baseline values. In the zero-contrast PCI group, serum creatinine levels did not change until 1 month, and were significantly increased at 1 year. Serum creatinine levels decreased in 32 out of 50 patients $(64 \%)$ at 1 day, 20 out of 49 patients $(40.8 \%)$ at 1 month, and 16 out of 46 patients $(34.8 \%)$ at 1 year. Serum creatinine levels decreased by $0.13(0.093-0.27)$ in 16 patients who had an improvement in renal function at 1 year (Supplementary Table).

\section{Discussion}

In the present study, we evaluated the effect of zero-contrast PCI on acute and long-term clinical outcomes in patients with stage 3-5 CKD and ischemic heart disease. Variation in renal function was also assessed using serum creatinine levels. The major findings of the present study are as follows: (1) all zero-contrast PCI procedures were successful without any complications; (2) renal events such as AKI and requirement of emergent hemodialysis did not occur during hospitalization; (3) the incidence of MACE was comparable between the zero-contrast and conven- 


\begin{tabular}{|c|c|c|c|}
\hline Angiographical characteristics & $\begin{array}{c}\text { Conventional } \mathrm{PCl} \\
(\mathrm{n}=85)\end{array}$ & $\begin{array}{c}\text { Zero-contrast PCI } \\
(n=72)\end{array}$ & $P$ value \\
\hline LMT lesion & $1(1.2)$ & $6(8.3)$ & 0.048 \\
\hline LAD lesion & $41(48.2)$ & $39(54.2)$ & 0.46 \\
\hline LCX lesion & $17(20.0)$ & $12(16.7)$ & 0.59 \\
\hline RCA lesion & $27(31.8)$ & $21(29.2)$ & 0.72 \\
\hline Diameter stenosis, \% & $90.0 \pm 6.7$ & $88.5 \pm 6.3$ & 0.091 \\
\hline Chronic total occlusion & $11(12.9)$ & $3(4.2)$ & 0.055 \\
\hline Lesion length $>20 \mathrm{~mm}$ & $27(31.8)$ & $28(38.9)$ & 0.35 \\
\hline Calcified lesion & $33(38.8)$ & $53(73.6)$ & $<0.0001$ \\
\hline Bifurcation lesion & $23(27.1)$ & $29(40.3)$ & 0.080 \\
\hline ACC/AHA class A & $20(23.5)$ & $7(9.7)$ & \multirow{4}{*}{0.15} \\
\hline ACC/AHA class B1 & $25(29.4)$ & 24 (33.3) & \\
\hline ACC/AHA class B2 & $8(9.4)$ & $9(12.5)$ & \\
\hline ACC/AHA class $\mathrm{C}$ & $32(37.7)$ & $32(44.4)$ & \\
\hline \multicolumn{4}{|l|}{ Procedural characteristics } \\
\hline Successful strategy & $82(96.5)$ & $72(100)$ & 0.25 \\
\hline Trans radial approach & $72(84.7)$ & $61(84.7)$ & 1.00 \\
\hline Trans femoral approach & $15(17.7)$ & $13(16.7)$ & 0.95 \\
\hline Guiding catheter size, $\mathrm{Fr}$ & $6.14 \pm 0.41$ & $6.04 \pm 0.20$ & 0.083 \\
\hline Procedure time, $\min$ & $43(32-67)$ & $51(38-67)$ & 0.19 \\
\hline Radiation dose, mGy & $472(296.0-651.9)$ & 276 (191.7-455.5) & $<0.0001$ \\
\hline Radiation duration, min & $14.7(7.5-26.8)$ & $19.3(12.8-28.6)$ & 0.046 \\
\hline Contrast volume (CAG), $\mathrm{mL}$ & $42.6(37-57)$ & $19.7(17.7-26.3)$ & $<0.0001$ \\
\hline Contrast volume (PCI), $\mathrm{mL}$ & $80.8(62.9-115.5)$ & $4.3(3.5-5.3)$ & $<0.0001$ \\
\hline \multicolumn{4}{|l|}{ Device } \\
\hline Number of guide wires & $1.64 \pm 0.10$ & $1.88 \pm 0.11$ & 0.10 \\
\hline Number of stents & $1.27 \pm 0.070$ & $1.14 \pm 0.076$ & 0.21 \\
\hline Total stent length, $\mathrm{mm}$ & $26.7 \pm 1.89$ & $27.7 \pm 2.13$ & 0.72 \\
\hline Stent diameter, $\mathrm{mm}$ & $2.98 \pm 0.054$ & $3.00 \pm 0.061$ & 0.80 \\
\hline Pre-dilatation & $56(65.9)$ & $45(62.5)$ & 0.66 \\
\hline Post-dilatation & 45 (52.9) & $34(47.2)$ & 0.48 \\
\hline $\mathrm{MLA}, \mathrm{mm}^{2}$ & $1.91 \pm 0.81$ & $2.00 \pm 0.61$ & 0.49 \\
\hline $\mathrm{MSA}, \mathrm{mm}^{2}$ & $6.01 \pm 1.82$ & $6.05 \pm 1.42$ & 0.86 \\
\hline
\end{tabular}

Data are presented as $n(\%)$, mean $\pm S D$, and/or median (Q1-Q3). ACC/AHA, American Heart Association/American College of Cardiology; CAG, coronary angiography; Calcified lesion, moderate or severe calcification on angiography; LAD, left anterior descending artery; LCX, left circumflex artery; LMT, left main trunk artery; MLA, minimum lumen area; MSA, minimum stent area; $\mathrm{PCl}$, percutaneous coronary intervention; RCA, right coronary artery.

tional PCI groups; (4) in long-term follow up, the incidence of renal replacement therapy was low after zero-contrast PCI; and (5) the serum creatinine level of patients with CKD significantly increased at 1 year; however, one-third of patients showed improvement of renal function after zero-contrast PCI and the effect was persistent at 1 year.

Very recently, a systematic review regarding the safety and efficacy of minimum- or zero-contrast PCI in patients with CKD with CCS was published. ${ }^{19}$ Their search did not find evidence regarding zero-contrast PCI, except for 2 studies, which had only short-term follow up without a control group. ${ }^{17,18}$ To the best of our knowledge, this is the first report to assess the long-term feasibility, safety, and efficacy of zero-contrast PCI in patients with CKD. The present study also assessed the possibility of renal function improvement by zero-contrast PCI in some patients with CKD.

\section{Previous Studies}

There is considerable evidence that contrast-associated
AKI is related to a worse prognosis after PCI. Contrastassociated AKI is more common and serious in patients with CKD. ${ }^{24}$ Prevention of contrast-associated AKI may be the key to improving prognosis after PCI, especially in patients with CKD. There is some evidence that PCI with minimal contrast prevents contrast-associated AKI.6-8 Thus, reducing contrast volume is believed to be the best strategy to prevent contrast-associated AKI and improve outcomes in patients with CKD. As Burlacu et al emphasized in their systematic review, ${ }^{19}$ recent guidelines on CCS recommend minimizing the use of iodinated contrast agents during PCI in patients with severe CKD and preserving urine production to prevent further deterioration (class I, level of evidence B). ${ }^{25}$

Zero-contrast PCI is the ultimate strategy in terms of preventing AKI in patients with CKD, although it is technically challenging. Limited evidence is available regarding the feasibility, safety, or efficacy of zero-contrast PCI. Ali et al investigated the impact of zero-contrast PCI 


\begin{tabular}{|lccc|}
\hline $\begin{array}{l}\text { Table 4. In-Hospital and Long-Term Outcomes for Patients in the Conventional PCI and Zero-Contrast PCI } \\
\text { Groups }\end{array}$ & $\begin{array}{c}\text { Conventional PCI } \\
(\mathbf{n}=50)\end{array}$ & $\begin{array}{c}\text { Zero-contrast PCI } \\
(\mathbf{n}=50)\end{array}$ & P value \\
$\begin{array}{l}909(390-1,340) \\
\text { Follow-up period (days) }\end{array}$ & $1,083(437-1,460)$ & 0.32 \\
In-hospital outcomes & $47(94.0)$ & $50(100)$ & 0.24 \\
Successful strategy & $1(2.0)$ & $0(0)$ & 1.0 \\
PCl complications & $0(0)$ & $0(0)$ & 1.0 \\
Acute kidney injury & $0(0)$ & $0(0)$ & 1.0 \\
Emergent hemodialysis & & & 0.78 \\
Long-term outcomes & $7(14.0)$ & $8(16.0)$ & 0.18 \\
MACE & $3(6.0)$ & $7(14.0)$ & 1.0 \\
All-cause death & $1(2.0)$ & $1(2.0)$ & 1.0 \\
Cardiovascular death & $0(0)$ & $0(0)$ & 0.17 \\
Non-fatal MI & $4(8.0)$ & $1(2.0)$ & 0.36 \\
Clinically driven TLR & $1(2.0)$ & $4(8.0)$ & \\
Renal replacement therapy & & & \\
\hline
\end{tabular}

Data are presented as $\mathrm{n}(\%)$, median (Q1-Q3). MACE, major cardiovascular death; MI, myocardial infarction; $\mathrm{PCl}$, percutaneous coronary intervention; $\mathrm{PCl}$ complications included coronary perforation, stent thrombosis and periprocedural myocardial infarction; TLR, target lesion revascularization.

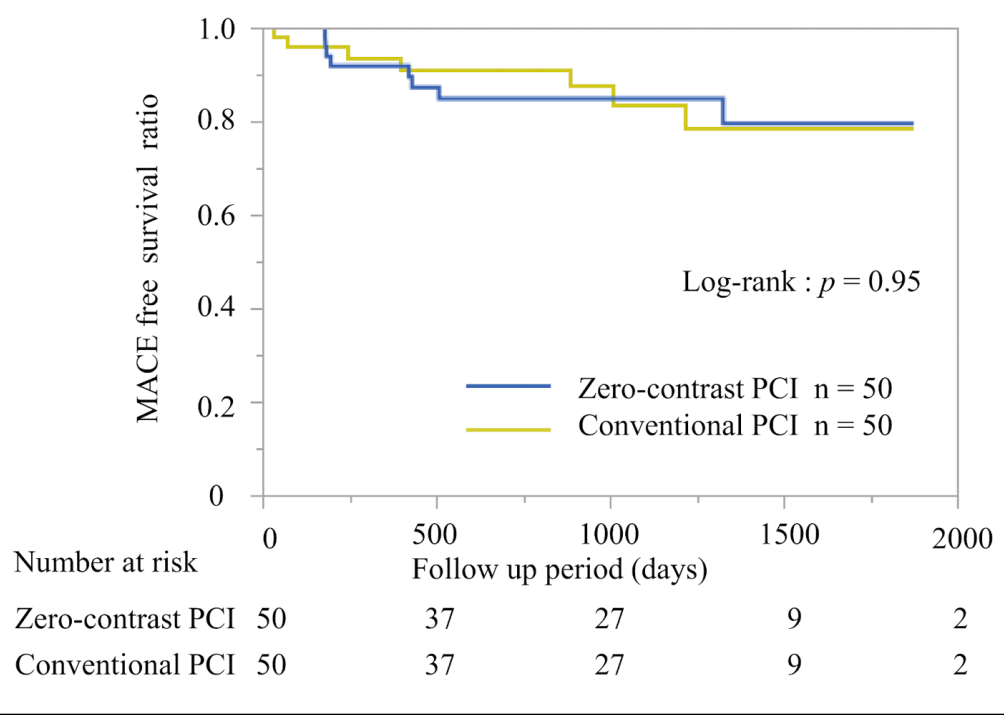

Figure 2. In the follow-up period, 8 patients experienced major adverse cardiovascular events (MACE) in the zero-contrast $\mathrm{PCl}$ group, whereas 7 patients experienced MACE in the conventional $\mathrm{PCl}$ group. There was no significant difference in Kaplan-Meier curves for MACE-free survival between the 2 groups (Log-rank, $\mathrm{P}=0.95$ )

on renal function and the need for renal replacement therapy in a total of 31 patients with CKD with a follow-up time of 79 days. ${ }^{17}$ Zero-contrast PCI was successful in all cases and resulted in no MACEs and preserved renal function without the need for renal replacement therapy. Although the study had a low number of patients, short-term outcomes, and a single arm without a control group, these findings make zero-contrast PCI a more attractive and promising strategy.

\section{Feasibility, Safety, and Use of Zero-Contrast PCI}

Patients with CKD form a high-risk group in terms of PCI procedures and long-term cardiovascular events. ${ }^{3}$ The population of the present study had conditions that included multi-vessel disease, left main trunk, calcified lesions, and complex lesions such as bifurcation or CTO. Even in highrisk patients, zero-contrast PCI was successful in all cases with optimal revascularization without contrast use. There were no procedural complications, such as persistent coronary dissection or hematoma, peri-procedural MI, or wire perforation. Furthermore, there were no renal events, such as AKI or emergent hemodialysis. Thus, the results of the present study support the feasibility and safety of elective zero-contrast PCI for patients with CKD. In the long-term follow up, with an average of 32 months, 6 patients experienced non-cardiovascular death and 1 patient experienced cardiovascular death. There was 1 target lesion revascularization and no non-fatal MI. The incidence of MACE in the zero-contrast PCI group was comparable to that of the conventional PCI group, which consisted mostly of patients without CKD. Thus, the present study also supports the long-term safety and use of zero-contrast PCI.

There were 4 events of renal replacement therapy in the long-term follow up after zero-contrast PCI. Among the 4 


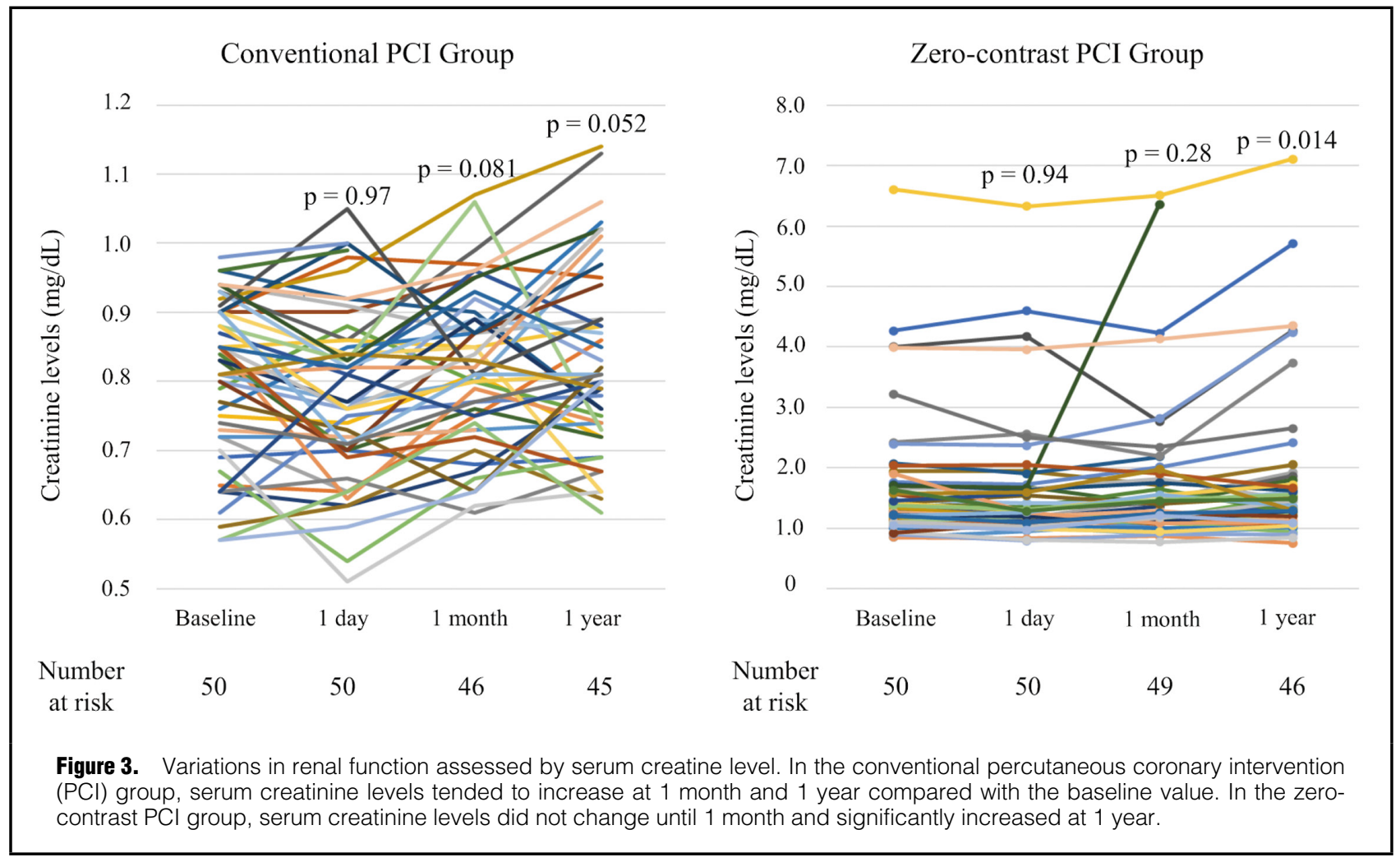

patients, 3 patients had stage 5 CKD and 1 patient had stage 4 CKD before the zero-contrast PCI procedure. The rate of dialysis initiation has been reported to be $0.6 \%$ for stage 3 CKD, $11.4 \%$ for stage 4 CKD, and $61.1 \%$ for stage $5 \mathrm{CKD}$ for 2 years (during an observation period of 22.6 \pm 11.9 months). ${ }^{26}$ In the present study population, the incidence of dialysis initiation was lower than that over the natural course of CKD. More than one-third of patients showed improved renal function even 1 year after zerocontrast PCI. Thus, zero-contrast PCI is not only safe, but also has some potential benefit to improve renal function in patients.

The results of the present study support the safety of zero-contrast PCI; however, there may be some unsuitable lesions that can be treated using this procedure. A heavy calcified and curved lesion, which is an extremely high-risk lesion that has a rotational atherectomy planned as treatment, may not be suitable for zero-contrast PCI. Procedural complications such as perforation or dissection are more serious effects compared with contrast-associated AKI. The strategy should be changed to mini-contrast PCI in some cases. The CTO lesion, which requires a tip injection or micro-channel angiography, may not be suitable for zero-contrast PCI because lesions treated with angiography need a small amount of contrast to increase the success rate of the CTO-PCI. Thus, case selection for zerocontrast PCI or mini-contrast PCI is important.

Even if the amount of contrast used was low, it might affect the incidence of contrast-associated AKI. Although the time interval between diagnostic coronary angiography and PCI was at least 10 days in the present study, it may be better to wait more than 1 month to prevent contrastassociated AKI, as Bugani et al recommended in their review. ${ }^{27}$

\section{Benefit of Zero-Contrast PCI for Renal Function}

It is unknown whether zero-contrast PCI improves renal function in patients with CKD and ischemic heart disease. Recently, we reported a case of ischemic cardiomyopathy who was scheduled to start hemodialysis for end-stage diabetic nephropathy but exhibited improved renal function after PCI with an extremely low contrast dose. ${ }^{23}$ The patient did not require dialysis $>2$ years after revascularization. Intrinsic renal failure due to injured renal parenchyma in conjunction with diabetes, hypertension, or chronic glomerulonephritis is irreversible. However, the cause of renal insufficiency should be multifactorial in some patients. If the cause of renal insufficiency is mainly ischemic cardiomyopathy, renal function may be partly reversible in accordance with left ventricular function after revascularization without contrast media. The median serum creatinine level significantly increased 1 year after zero-contrast PCI due to primary CKD; however, the serum creatinine level decreased even at 1 year in more than one-third of the patients in the present study. The results of the present study suggest the possibility that zero-contrast PCI may improve renal function in a specific population.

The ISCHEMIA-CKD trial demonstrated that an initial invasive strategy did not improve the clinical outcomes in patients with ischemic heart disease and CKD compared to an initial conservative strategy; ${ }^{5}$ however, in the trial, the prevention of contrast-associated AKI and optimal stent implantation by IVUS-guided zero-contrast PCI were not assessed. Zero-contrast PCI may improve the clinical outcomes in patients with ischemic heart disease and CKD compared to an initial conservative strategy.

\section{Study Limitations}

This study had several limitations. First, this is a small, 
non-randomized, single-center study. Second, although we tried to adjust for confounding factors via propensity score matching, we cannot exclude the possibility of residual contributing factors as a result of the presence of an unmeasured confounder or measurement errors in the included factors. Third, although the follow-up period was long, with an average of 32 months, the number of cardiovascular events was low and may be underpowered to compare the 2 groups. Fourth, although the contrast volume was reduced for coronary angiography, it may have affected clinical outcomes. Fifth, to investigate the possible benefit of zero-contrast PCI on contrast-associated AKI, it is better to assess the more sensitive bio-marker for AKI, such as urinary L-FABP (Liver-type Fatty AcidBinding Protein) in addition to serum creatinine level in the prospective study. Sixth, presence of proteinuria is one of the important values for the diagnosis of CKD; however, the data about proteinuria was not available in the registry. Finally, zero-contrast PCI is challenging and requires special expertise and experience; thus, the results of the present study may not be generalizable to other populations. Further studies are needed to confirm the safety and use of zero-contrast PCI.

\section{Conclusions}

In this study, all zero-contrast PCI procedures were successful without any procedural complications in patients with CKD. The incidence of MACE was comparable between patients with CKD treated with zero-contrast PCI and patients without CKD treated with conventional PCI. Renal events did not occur during the hospital course, and the incidence of renal replacement therapy was low after zero-contrast PCI, suggesting the benefit of zero-contrast PCI for renal function and clinical outcomes.

\section{Acknowledgments}

None.

\section{Disclosures}

The authors declare no conflicts of interest.

\section{IRB Information}

The present study was approved by the Showa University Koto-Toyosu Hospital Institutional Review Board for Clinical Research (Reference number: 21T7012).

\section{References}

1. Manjunath G, Tighiouart H, Ibrahim H, MacLeod B, Salem DN, Griffith JL, et al. Level of kidney function as a risk factor for atherosclerotic cardiovascular outcomes in the community. $J$ Am Coll Cardiol 2003; 41: 47-55.

2. McCullough PA, Soman SS, Shah SS, Smith ST, Marks KR, Yee $\mathrm{J}$, et al. Risks associated with renal dysfunction in patients in the coronary care unit. J Am Coll Cardiol 2000; 36: 679-684.

3. Best PJM, Lennon R, Ting HH, Bell MR, Rihal CS, Holmes $\mathrm{DR}$, et al. The impact of renal insufficiency on clinical outcomes in patients undergoing percutaneous coronary interventions. $J$ Am Coll Cardiol 2002; 39: 1113-1119.

4. Hashimoto Y, Ozaki Y, Kan S, Nakao K, Kimura K, Ako J, et al. Impact of chronic kidney disease on in-hospital and 3-year clinical outcomes in patients with acute myocardial infarction treated by contemporary percutaneous coronary intervention and optimal medical therapy: Insights from the J-MINUET Study. Circ J 2021; 85: 1710-1718.

5. Bangalore S, Maron DJ, O'Brien SM, Fleg JL, Kretov EI, Briguori $\mathrm{C}$, et al. Management of coronary disease in patients with advanced kidney disease. $N$ Engl J Med 2020; 382: 1608-1618.
6. Ledru F, Théroux $\mathrm{P}$, Lespérance $\mathrm{J}$, Laurier $\mathrm{J}$, Ducimetière $\mathrm{P}$, Guermonprez JL, et al. Geometric features of coronary artery lesions favoring acute occlusion and myocardial infarction: A quantitative angiographic study. J Am Coll Cardiol 1999; 33: $1353-1361$.

7. Mehran R, Aymong ED, Nikolsky E, Lasic Z, Iakovou I, Fahy $\mathrm{M}$, et al. A simple risk score for prediction of contrast-induced nephropathy after percutaneous coronary intervention: Development and initial evaluation. J Am Coll Cardiol 2004; 44: 13931399.

8. James MT, Samuel SM, Manning MA, Tonelli M, Ghali WA, Faris $\mathrm{P}$, et al. Contrast-induced acute kidney injury and risk of adverse clinical outcomes after coronary angiography: A systematic review and meta-analysis. Circ Cardiovasc Interv 2013; 6: 37-43.

9. Marenzi G, Assanelli E, Campodonico J, Lauri G, Marana I, De Metrio M, et al. Contrast volume during primary percutaneous coronary intervention and subsequent contrast-induced nephropathy and mortality. Ann Intern Med 2009; 150: 170-177.

10. Kang SJ, Mintz GS, Park DW, Lee SW, Kim YH, Whan Lee C, et al. Mechanisms of in-stent restenosis after drug-eluting stent implantation: Intravascular ultrasound analysis. Circ Cardiovasc Interv 2011; 4: 9-14.

11. Fitzgerald PJ, Oshima A, Hayase M, Metz JA, Bailey SR, Baim DS, et al. Final results of the Can Routine Ultrasound Influence Stent Expansion (CRUISE) study. Circulation 2000; 102: $523-$ 530.

12. Mariani J Jr, Guedes C, Soares P, Zalc S, Campos CM, Lopes $\mathrm{AC}$, et al. Intravascular ultrasound guidance to minimize the use of iodine contrast in percutaneous coronary intervention: The MOZART (minimizing contrast utilization with IVUS guidance in coronary angioplasty) randomized controlled trial. JACC Cardiovasc Interv 2014; 7: 1287-1293.

13. Kane GC, Doyle BJ, Lerman A, Barsness GW, Best PJ, Rihal CS. Ultra-low contrast volumes reduce rates of contrast-induced nephropathy in patients with chronic kidney disease undergoing coronary angiography. J Am Coll Cardiol 2008; 51: 89-90.

14. Ogata N, Ikari Y, Nanasato M, Okutsu M, Kametani R, Abe M, et al. Safety margin of minimized contrast volume during percutaneous coronary intervention in patients with chronic kidney disease. Cardiovasc Interv Ther 2014; 29: 209-215.

15. Ebisawa S, Kurita T, Tanaka N, Nasu K, Kimura M, Ito T, et al. Impact of minimum contrast media volumes during elective percutaneous coronary intervention for prevention of contrastinduced nephropathy in patients with stable coronary artery disease. Cardiovasc Interv Ther 2016; 31: 13-20.

16. Kurogi K, Ishii M, Sakamoto K, Komaki S, Kusaka H, Yamamoto $\mathrm{N}$, et al. Optical coherence tomography-guided percutaneous coronary intervention with low-molecular-weight dextran: Effect on renal function. Circ J 2020; 84: 917-925.

17. Ali ZA, Karimi Galougahi K, Nazif T, Maehara A, Hardy MA, Cohen DJ, et al. Imaging- and physiology-guided percutaneous coronary intervention without contrast administration in advanced renal failure: A feasibility, safety, and outcome study. Eur Heart J 2016; 37: 3090-3095.

18. Sacha J, Gierlotka M, Lipski P, Feusette P, Dudek D. Zerocontrast percutaneous coronary interventions to preserve kidney function in patients with severe renal impairment and hemodialysis subjects. Postepy Kardiol Interwencyjnej 2019; 15: 137-142.

19. Burlacu A, Tinica G, Brinza C, Crisan-Dabija R, Popa IV, Covic A. Safety and efficacy of minimum- or zero-contrast IVUSguided percutaneous coronary interventions in chronic kidney disease patients: A systematic review. J Clin Med 2021; 10: 1996.

20. Thygesen K, Alpert JS, Jaffe AS, Chaitman BR, Bax JJ, Morrow DA, et al; Executive Group on behalf of the Joint European Society of Cardiology (ESC)/American College of Cardiology (ACC)/ American Heart Association (AHA)/World Heart Federation (WHF) Task Force for the Universal Definition of Myocardial Infarction. Fourth Universal Definition of Myocardial Infarction (2018). J Am Coll Cardiol 2018; 72: 2231-2264.

21. Mintz GS. Intravascular ultrasound guidance improves patient survival (mortality) after drug-eluting stent implantation: Review and updated bibliography. Cardiovasc Interv Ther 2020; 35: $37-43$.

22. Saito Y, Kobayashi Y, Fujii K, Sonoda S, Tsujita K, Hibi K, et al. Clinical expert consensus document on standards for measurements and assessment of intravascular ultrasound from the Japanese Association of Cardiovascular Intervention and Therapeutics. Cardiovasc Interv Ther 2020; 35: 1-12.

23. Shibata K, Wakabayashi K, Kosaki R, Sato C, Nishikura T, Shinke T, et al. Ultra-minimum contrast percutaneous coronary 
intervention for a patient with complex coronary artery disease and end-stage diabetic nephropathy. J Cardiol Cases 2021; 23: 290-293.

24. Giacoppo D, Madhavan MV, Baber U, Warren J, Bansilal S, Witzenbichler B, et al. Impact of contrast-induced acute kidney injury after percutaneous coronary intervention on short- and long-term outcomes: Pooled analysis from the HORIZONS-AMI and ACUITY trials. Circ Cardiovasc Interv 2015; 8: e002475.

25. Knuuti J, Wijns W, Saraste A, Capodanno D, Barbato E, FunckBrentano C, et al. 2019 ESC Guidelines for the diagnosis and management of chronic coronary syndromes. Eur Heart J 2020; 41: $407-477$.

26. Nakayama M, Sato T, Miyazaki M, Matsushima M, Sato H,
Taguma Y, et al. Increased risk of cardiovascular events and mortality among non-diabetic chronic kidney disease patients with hypertensive nephropathy: The Gonryo study. Hypertens Res 2011; 34: 1106-1110.

27. Bugani G, Ponticelli F, Giannini F, Gallo F, Gaudenzi E, Laricchia A, et al. Practical guide to prevention of contrast-induced acute kidney injury after percutaneous coronary intervention. Catheter Cardiovasc Interv 2021; 97: 443-450.

\section{Supplementary Files}

Please find supplementary file(s);

http://dx.doi.org/10.1253/circj.CJ-21-0905 